\title{
Spacelike self-similar shrinking solutions of the mean curvature flow in pseudo-Euclidean spaces
}

\author{
Márcio Rostirolla Adames
}

\begin{abstract}
I classify spacelike self-similar shrinking solutions of the mean curvature flow in pseudo-Euclidean space in arbitrary codimension, if the mean curvature vector is not a null vector and the principal normal vector is parallel in the normal bundle. Moreover, I exclude the existence of such self-shrinkers in several cases. The classification is analogous to the existing classification in the Euclidean case [20, 27].
\end{abstract}

\section{Introduction}

The mean curvature flow (MCF) of an immersion $F: M \rightarrow N$ of a smooth manifold $M$ into a Riemannian manifold $(N, h)$ is a natural way to deform this immersion into something "rounder" or "more regular." It is a smooth family of isometric immersions $F_{t}: M \rightarrow N, t \in[0, T)$ that satisfies

$$
\frac{d F_{t}}{d t}=\vec{H}, \quad F_{0}(x)=F(x) .
$$

The MCF has been studied by many. It not always regularizes the initial surface but also produces singularities. Suppose now that the target manifold $N$ is the Euclidean space $\mathbb{E}^{n}$. In an important work on the MCF of convex compact hypersurfaces [18] Huisken showed, among other results, that the supremum of the norm of the second fundamental form $\sup _{M}\|A\|^{2}$ explodes as $t \rightarrow T$ (the maximal existence time) if there is a finite time $(T<\infty)$ singularity. However, this can be done not only for hypersurfaces, but also for a broader class of manifolds and in any codimension (see [26] Proposition 3.11 and Remark 3.12 or [12]).

In a subsequent work [19], Huisken showed, with his famous monotonicity formula, that hypersurfaces satisfying a natural growth in the norm of the second fundamental form, deform asymptotically near a singularity to self-similar solutions of the MCF after some blow up process (rescaling the surface and changing the time variable). Later Ilmanen [22] and White [30] 
proved that the all finite time singularities in the generalized sense of the Brakke flow [7] are self-similar solutions of the MCF.

These self-similar solutions of the MCF are also called self-shrinkers to avoid confusion with other types of solutions that preserve the "form" of the surface, like self-expanders and translating solutions. They are homotheties that shrink the initial manifold and are characterized by the equation

$$
\vec{H}=-F^{\perp} .
$$

Because of the relation between singularities of the MCF and selfshrinkers, there is interest in classifying and giving examples of these in special cases. Abresch and Langer [1] gave the complete classification of the closed plane curves that shrink homothetically, they are the circles and the so called Abresch \& Langer curves. Huisken proved in [20] that the self-shrinking hypersurfaces with non-negative mean curvature (compact or non-compact) are spheres, cylinders and the product of an Abresch \& Langer curve with an affine space. The result of Huisken was later generalized by Smoczyk [27] for higher codimensional immersions, with the assumption that the principal normal is parallel in the normal bundle and $\|\vec{H}\|_{\mathbb{E}} \neq 0$. A related result was found by Cao and $\mathrm{Li}[8]$ in any codimension: the selfshrinkers with $\|A\|^{2} \leq 1$ are spheres, planes or cylinders. There are also Bernstein type results for self-shrinkers in higher codimension of Ding and Wang [13], who generalize works of Wang [28] and Ecker and Huisken [15]. Recently, Baker [5] proved that high codimensional self-shrinkers under certain conditions for the second fundamental tensor and mean curvature vector are spheres or cylinders.

For hypersurfaces in $\mathbb{R}^{3}$, there are examples of a shrinking doughnut of Angenent [4] and many numerical examples of Chopp [9] and Ilmanen [21], like "punctured saddles" made of many handles crushing at the same time, which are highly unstable, depending on the surface having many symmetries. Colding and Minicozzi [11] showed that the only stable selfshrinkers of the MCF for initial smooth closed embedded surfaces in $\mathbb{R}^{3}$ are cylinders and spheres. For the Lagrangian MCF, Joyce et al. [23], Anciaux [3] and Wang [29] have examples. There are other results in different contexts.

The main purpose of this work is to study self-shrinkers of the MCF in higher codimension in the pseudo-Euclidean case. By that, we mean that the target manifold $N$ is a pseudo-Euclidean space, so that the most interesting new case is the Minkowski space $\mathbb{R}^{1, n}$. The MCF of spacelike hypersurfaces in the Minkowski space was studied for example by Ecker [14] and a related flow was considered by Ecker and Huisken [16]. Gerhardt [17] also studies 
curvature flows in semi-Riemannian manifolds, specially the inverse MCF. Beyond this, Bergner and Schäfer [6] considered the MCF in the threedimensional Minkowski space. Furthermore Li and Salavessa [24] have some results for the MCF of spacelike graphs in product manifolds.

The present paper is organized in the following fashion: in Section 2 are given some fundamental equations in order to fix the notation and in Section 3, we consider homotheties of the MCF that lie in hyperquadrics, and find, similarly to Smoczyk's result in [27] for spheres in the Euclidean space $\mathbb{E}^{n}$, that the homotheties of the MCF with initial immersion contained in a hyperquadric $\left(\left\{X \in \mathbb{R}^{p, n} \mid\|X\|^{2}=k\right\}\right)$ are exactly the minimally immersed submanifolds of the hyperquadric if $k>0$ or $k<0$, as Theorems 3.5 and 3.6 state. Moreover, given the initial minimal immersion, the flow can be explicitly calculated. If $k=0$ (the light cone), a homothety with non-degenerate first fundamental form would immediately leave the light cone and thence could not be a homothety starting at $t=0$ because the light cone is star shaped, as stated in Theorem 3.7. But, as Ecker noted in [14], the upper light cone would immediately change to a hyperquadric and flow homothetically after $t=0$.

There is a relevant difference between the flow of minimal surfaces of the hyperquadrics with $k>0$ and the ones with $k<0$ (which do not exist in Euclidean case); if $k>0$, they shrink to a point (at least the compact ones) in finite time, but if $k<0$, they expand and never produce singularities. Beyond this, they are given by different equations. The following results are for the shrinking ${ }^{1}$ case, which are the isometric immersions $F: M \rightarrow$ $\left(\mathbb{R}^{n},\langle\cdot, \cdot\rangle\right)$ satisfying

$$
\vec{H}=-F^{\perp} .
$$

If one considers the self-shrinkers and self-expanders that are contained in the hyperquadrics as submanifolds in the pseudo-Euclidean space $\left(\mathbb{R}^{n}\right.$, $\langle\cdot, \cdot\rangle)$, then one observes that $\nabla^{\perp} \vec{H} \equiv 0$ and $\nabla^{\perp} \nu \equiv 0$, where $\nu:=\vec{H} /\|\vec{H}\|$ is the principal normal. A natural question is whether these conditions are also sufficient to guarantee that a spacelike ${ }^{2}$ self-shrinker lies in a hyperquadric. The condition $\nabla^{\perp} \vec{H}=0$ implies this immediately if $M$ is compact, because $\|\vec{H}\|^{2}$ is then constant and the maximum principle implies, with equation

$$
\triangle\|F\|^{2}=2 m-2\|\vec{H}\|^{2}
$$

\footnotetext{
${ }^{1}$ The expanding case satisfies $\vec{H}=F^{\perp}$.

${ }^{2}$ We use elliptic methods to obtain our results (maximum principles, that do not hold for hyperbolic equations).
} 
that $\|F\|^{2}$ is constant. So, in this work, are examined self-shrinkers of the MCF with $\|\vec{H}\|^{2} \neq 0$ and $\nabla^{\perp} \nu=0$. The condition $\nabla^{\perp} \nu=0$ is natural since it holds for hypersurfaces.

Section 4 deals with fundamental equations for self-shrinkers with the principal normal parallel in the normal bundle and the compact case. If $\|\vec{H}\|^{2}>0$ one finds, just as Smoczyk in [27] for the Euclidean case, that if $M$ is compact and $\operatorname{dim}(M) \geq 2$, the only spacelike self-shrinkers of the MCF with $\|\vec{H}\|^{2}(p) \neq 0, \forall p \in M$ and $\nabla^{\perp} \nu \equiv 0$ are the minimal ${ }^{3}$ submanifolds of hyperquadrics, as stated in Theorem 4.1. This dimensional restriction is in fact optimal because in dimension one there are the Abresch \& Langer curves which are self-shrinkers and do not lie in spheres. The result is proved controlling the quantity $\|P\|^{2} /\|\vec{H}\|^{4}$, which is scaling invariant, thence likely to be constant in an homothety. This quantity is related to the quantity $\|A\|^{2} /\|\vec{H}\|^{2}$ considered by Huisken, which is hard to control in high codimension. The problem (arising from the pseudo-Euclidean background) of not being able to tell the sign of $\|A\|^{2}$ and $\|\vec{H}\|^{2}$ is avoided because equations (and not inequalities) are found for the most important quantities.

Equation (1.1) already shows that there are no compact self-shrinkers with $\|\vec{H}\|^{2}<0$. In this paper, the inexistence of self-shrinkers with $\|\vec{H}\|^{2}<0$ is proven, also in the non-compact case under certain hypothesis, as stated in Theorem 7.4.

As a consequence, the Minkowski space does not (in all of our treated cases) have spacelike self-shrinking hypersurfaces, which can be surprising and is related Ecker's longtime existence result for spacelike hypersurfaces in Minkowski space [14].

Sections 5 to 7 are about the non-compact case. Again following Smoczyk in the non-compact case, one finds that the self-shrinkers with $\|\vec{H}\|^{2}>0$ are products of affine spaces with minimal submanifolds of hyperquadrics or with homothetic solutions of the curve shortening flow ${ }^{4}$ as stated in Theorem 1.1.

Theorem 1.1. Let $M$ be a smooth manifold and $F: M \rightarrow \mathbb{R}^{q, n}$ be a mainly positive, spacelike, shrinking self-similar solution of the MCF with bounded geometry such that $F(M)$ is unbounded. Beyond that, let $F$ satisfy the conditions: $\|\vec{H}\|^{2}(p) \neq 0$ for all $p \in M$ and the principal normal is parallel in

\footnotetext{
${ }^{3}$ By minimal we mean the ones satisfying $\vec{H}=0$. We use this name because the condition $\vec{H}=0$ is then mnemonic, although this condition does not imply minimality of the volume functional in pseudo-Euclidean spaces, so they are just critical points of the volume functional.

${ }^{4}$ The curve shortening flow is the MCF for plane curves.
} 
the normal bundle $\left(\nabla^{\perp} \nu \equiv 0\right)$. Then one of the two holds

$$
\begin{aligned}
& F(M)=\mathcal{H}_{r} \times \mathbb{R}^{m-r} \quad \text { or } \\
& F(M)=\Gamma \times \mathbb{R}^{m-1},
\end{aligned}
$$

where $\mathcal{H}_{r}$ is an $r$-dimensional minimal surface of the hyperquadric $\mathcal{H}^{n-1}(r)$ (in addition $\|\vec{H}\|^{2}=r>0$ ) and $\Gamma$ is a rescaling of an Abresch \& Langer curve in a spacelike plane. By $R^{m-r}$, we mean an $m-r$ dimensional spacelike affine space in $\mathbb{R}^{q, n}$.

The proof of this theorem is long and internally divided in lemmas to make its several steps easier to recognize. It was necessary to divide it in two cases (Theorems 6.1 and 7.1). In both of them, we split $T M$ into two involutive distributions. Then we use the Theorem of Frobenius to get foliations on $M$ whose leaves are totally geodesic immersed in $M$. After this, we calculate a formula that relates the second fundamental tensor of $F$ with these distributions. In particular, the second fundamental tensor of $F$ is zero when restricted to one of these distributions, so that the leaves of this distribution are totally geodesic in $\left(\mathbb{R}^{q, n},\langle\cdot, \cdot\rangle\right)$ and then, considering parallel transports inside these leaves, one finds that they are parallel affine subspaces of $\mathbb{R}^{q, n}$. The other distribution delivers the $\mathcal{H}_{r}$ and $\Gamma$ parts in the last theorem. We get this considering the second fundamental tensor and mean curvature vectors of the inclusion of the leaves related to this distribution, with some extra effort to prove that $\Gamma$ lies on a plane (based on an idea of [20]). In the last step, we construct an explicit map from these second leaves times $\mathbb{R}^{m-r}$ onto $F(M)$.

\section{Geometric background}

\subsection{Inner product spaces}

Let $\left(\mathbb{R}^{n},\langle\cdot, \cdot\rangle\right)$ be an inner product space. This means that $\langle\cdot, \cdot\rangle$ is a symmetric bilinear form which is non-degenerate.

A reference the subject is [25] by $\mathrm{O}^{\prime} \mathrm{Neill}$.

The index $\eta$ of an inner product $\langle\cdot, \cdot\rangle$ over $V$ is the maximum of the dimensions of subspaces $W \subset V$ on which $\left.\langle\cdot, \cdot\rangle\right|_{W}$ is negative definite.

Definition 2.1. Let $U \subset V$ be a vector subspace of $V$. The normal subspace $U^{\perp}$ is the set

$$
U^{\perp}:=\{v \in V:\langle v, u\rangle=0 \forall u \in U\}
$$


It is not always possible to decompose $V$ into $U \oplus U^{\perp}$, but the following holds:

Lemma 2.2. A subspace $U$ of $V$ is non-degenerate if, and only if, $V=$ $U \oplus U^{\perp}$.

And $U$ is non-degenerate if, and only if, $U^{\perp}$ is non-degenerate.

There are three kinds of vectors in inner product spaces:

Definition 2.3. Let $v \in V$ be a vector. $v$ is said to be

$$
\begin{array}{cc}
\text { spacelike } & \text { if }\langle v, v\rangle>0 \text { or } v=0, \\
\text { null } & \text { if }\langle v, v\rangle=0 \text { and } v \neq 0, \\
\text { timelike } & \text { if }\langle v, v\rangle<0 .
\end{array}
$$

We say that a vector subspace $W$ of $V$ is spacelike if all $w \in W$ are spacelike.

Definition 2.4. Let $V$ be a $n$-dimensional inner product space. A unit vector $v \in V$ is a vector such that $\|v\|^{2}= \pm 1$. A set of $k$ mutually orthogonal unit vectors is said to be orthonormal.

Lemma 2.5. An inner product space $V \neq 0$ has an orthonormal basis.

Vectors can be written in this orthonormal basis in a unique way: if $\left\{e_{1}, \ldots, e_{n}\right\}$ is an orthonormal basis for $V$ with $\varepsilon_{i}:=\left\langle e_{i}, e_{i}\right\rangle$. Then, for $v \in V$, it holds

$$
v=\sum_{i=1}^{n} \varepsilon_{i}\left\langle v, e_{i}\right\rangle e_{i} .
$$

It is possible to generalize the notion of inner product space to differentiable manifolds.

Definition 2.6. Let $M$ be a differentiable manifold and $\langle\cdot, \cdot\rangle \in \Gamma\left(T^{*} M \otimes\right.$ $\left.T^{*} M\right)$ be a smooth section such that $\langle\cdot, \cdot\rangle(x)$ is an inner product for each $x \in M$. Then $\langle\cdot, \cdot\rangle$ is called a semi-Riemannian metric over $M$ and the pair $(M,\langle\cdot, \cdot\rangle)$ is called a semi-Riemannian manifold.

Let $N$ be a differentiable manifold, $F: N \rightarrow M$ be an immersion and $g:=F^{*}\langle\cdot, \cdot\rangle$ the first fundamental form of $N$. If $g$ is non-degenerate $N$ is also a semi-Riemannian manifold. $N$ is said to be spacelike if $g$ is a Riemannian metric on $N$. 
Definition 2.7. For $n \in\{2,3, \ldots\}$, we call the set

$$
\mathcal{H}^{n-1}(k):=\left\{x \in \mathbb{R}^{n}:\|x\|^{2}=k\right\}
$$

Hyperquadric of dimension $n-1$ and parameter $k, k \in \mathbb{R}$ fixed.

If $k \neq 0$ then $\mathcal{H}^{n-1}(k)$ is a smooth manifold and $\mathcal{H}^{n-1}(0) \backslash\{0\}$ is a smooth manifold.

\subsection{Differential geometry}

Let $F: M \rightarrow\left(\mathbb{R}^{n},\langle\cdot, \cdot\rangle\right)$ an immersion. The identification of $T_{p} \mathbb{R}^{n}$ with $\mathbb{R}^{n}$ induces a semi-Riemannian metric (denoted also by $\langle\cdot, \cdot\rangle$ ) on $T_{p} \mathbb{R}^{n}$ and the immersion $F$ induces a semi-Riemannian metric $g:=F^{*}\langle\cdot, \cdot\rangle$ over $M$, if it is non-degenerate. We assume that $g$ is non-degenerate. Let $\nabla^{g}$ be the Levi-Civita connection induced by $g$. Then

$$
d F\left(\nabla_{X}^{g} Y\right)=\left(D_{d F(X)} d F(Y)\right)^{\top} .
$$

Remark 2.8. We write $A$ and $\vec{H}$ (sometimes $A_{F}$ or $\vec{H}_{F}$ ) for the second fundamental tensor and the mean curvature vector of an isometric immersion $F$.

We use Latin letters for indices of tensors on $M$ and Greek letters for indices of tensors on $\left(\mathbb{R}^{n},\langle\cdot, \cdot\rangle\right)$. We also use the Einstein's convention for sums

$$
A_{i j}=\frac{\partial^{2} F^{\alpha}}{\partial x^{i} \partial x^{j}} \frac{\partial}{\partial y^{\alpha}}-\frac{\partial F^{\alpha}}{\partial x^{k}} \Gamma_{i j}^{k} \frac{\partial}{\partial y^{\alpha}}=\nabla_{i} \nabla_{j} F
$$

considering $F \in \Gamma\left(F^{*} T \mathbb{R}^{n}\right)$.

The (rough) Laplacian $\triangle$ is used on sections of several bundles and written $\triangle:=g^{i j} \nabla_{i} \nabla_{j}$ and $\triangle^{\perp}:=g^{i j} \nabla_{i}^{\perp} \nabla_{j}^{\perp}$. It follows that $\vec{H}=\triangle F$.

The following convention for the Riemannian curvature is adopted:

$$
\begin{aligned}
R_{k i j}^{l} \frac{\partial}{\partial x^{l}} & =R\left(\frac{\partial}{\partial x^{i}}, \frac{\partial}{\partial x^{j}}\right) \frac{\partial}{\partial x^{k}} \quad \text { and } \quad R_{s k i j}=R\left(\frac{\partial}{\partial x^{s}}, \frac{\partial}{\partial x^{k}}, \frac{\partial}{\partial x^{i}}, \frac{\partial}{\partial x^{j}}\right) \\
& =R_{k i j}^{l} g_{l s} .
\end{aligned}
$$

The Codazzi equation in local coordinates is written as

$$
\nabla_{l} A_{i j}-\nabla_{i} A_{l j}=R_{j l i}^{k} F_{k}^{\alpha}
$$


and considering $A$ as a section in the normal bundle, $\nabla_{l}^{\perp} A_{i j}-\nabla_{i}^{\perp} A_{l j}=$ $\left[R_{j l i}^{k} F_{k}^{\alpha}\right]^{\perp}=0$.

We make use of Gauß equation

$$
R_{k l i j}=\left\langle A_{i k}, A_{j l}\right\rangle-\left\langle A_{j k}, A_{i l}\right\rangle
$$

and the Ricci equation

$$
R^{\perp}(X, Y) \eta=\operatorname{tr}(\langle\eta, A(Y, \cdot)\rangle A(X, \cdot))-\operatorname{tr}(\langle\eta, A(X, \cdot)\rangle A(Y, \cdot))
$$

The Riemannian curvature tensor of the normal bundle $R_{i j}^{\perp}$ can be seen as the section $\left\langle R^{\perp}\left(\frac{\partial}{\partial x^{i}}, \frac{\partial}{\partial x^{j}}\right) \cdot, \cdot\right\rangle \in \Gamma\left(T^{*} M^{\perp} \otimes T^{*} M^{\perp}\right)$. The Ricci equation is then written

$$
R_{i j}^{\perp}=A_{j k} \otimes A_{i}^{k}-A_{i k} \otimes A_{j}^{k}=: A_{j k} \wedge A_{i}^{k}
$$

Furthermore we use the commutation formula, that can be found in [10].

\section{Hyperquadric homotheties of the MCF}

\subsection{Hyperquadric homotheties of the MCF}

In this section, we prove that the minimal immersions of the hyperquadrics deform homothetically under the MCF and that these are the only submanifolds of the hyperquadrics that do so.

Definition 3.1. Let $M$ be a smooth manifold, $(N, h)$ be a semi-Riemannian manifold and $F_{0}: M \rightarrow N$ be an immersion. A smooth a family of isometric immersions $F: M \times[0, T) \rightarrow N$, for some $T>0$, such that the metric $g_{t}:=$ $F(\cdot, t)^{*} h$ is non-degenerate for all $t \in[0, T)$ is called a solution of the $M C F$ with initial immersion $F_{0}$ if it satisfies

$$
\text { (3.1) } \quad \frac{d F}{d t}(p, t)=\vec{H}(p) \quad \text { and } \quad F(p, 0)=F_{0}(p) \forall p \in M, t \in[0, T) \text {, }
$$

where $\vec{H}$ is the mean curvature vector of the immersion $F(\cdot, t): M \rightarrow(N, h)$.

Now we consider properties of homotheties generated by the MCF. Let $F: M \times[0, T) \rightarrow\left(\mathbb{R}^{n},\langle\cdot, \cdot\rangle\right)$ be a solution of the MCF for some initial immersion, such that there is a rescaling function $c:[0, T) \rightarrow(0, \infty)$, with $c(0)=1$, 
so that $\tilde{F}:=c F$ satisfies

$$
\left\langle\frac{d \tilde{F}}{d t}(p, t), \tilde{\nu}\right\rangle=0, \quad \forall \tilde{\nu} \in T_{p} M^{\perp}
$$

which implies

$$
\vec{H}=-\frac{\dot{c}}{c} F^{\perp}
$$

In particular, we look now at the hyperquadric homotheties of the MCF in $\left(\mathbb{R}^{n},\langle\cdot, \cdot\rangle\right)$ and start recalling that if such a homothety starts in a hyperquadric, it stays in hyperquadrics:

Lemma 3.2. If $F(0, x) \subset \mathcal{H}^{n-1}(k(0))$ for all $x \in M$ then $F(t, x) \subset \mathcal{H}^{n-1}$ $(k(t))$ for all $x \in M$, for some function $k:[0, T) \rightarrow \mathbb{R}$.

As the position vector in a hyperquadric is normal to the hyperquadric, it follows that $F$ is always orthogonal to $\mathcal{H}^{n-1}(k(t))$. With this, $\|F\|^{2}$ can be calculated:

Lemma 3.3. $\|F(t)\|^{2}=k(0)-2 m t$ for $t \in[0, T)$.

We prove now that a hyperquadric homothety of the MCF is a minimal immersion in the hyperquadric $\mathcal{H}^{n-1}(k(0)-2 m t)$ for all $t \in[0, T)$. We will need the following rule for the composition of immersions: let $F: M \rightarrow N$ and $G: N \rightarrow P$ be isometric immersions between semi-Riemannian manifolds $(M, g),(N, h)$ and $(P, l)$. Denote $\vec{H}_{F}, \vec{H}_{G}$ and $\vec{H}_{G \circ F}$ the mean curvatures of $F, G$ and $G \circ F$, respectively. Then

$$
(\nabla d(G \circ F))_{x}=(\nabla d G)_{F(x)}(d F \cdot, d F \cdot)+d G_{F(x)} \circ \nabla d F
$$

and $\vec{H}_{G \circ F}=d G\left(\vec{H}_{F}\right)+\operatorname{tr}_{M}(\nabla d G)(d F \cdot, d F \cdot)$.

Theorem 3.4. Let $F: M \times[0, T) \rightarrow\left(\mathbb{R}^{n},\langle\cdot, \cdot\rangle\right)$ be a hyperquadric homothety of the $M C F$, then $F(M, t)$ is a minimal surface of the hyperquadric $\mathcal{H}^{n-1}\left(\|F(0)\|^{2}-2 m t\right)$ for all $t \in[0, T)$.

Proof. We consider the natural inclusion $I(t)$ of the hyperquadric $\mathcal{H}^{n-1}$ $(k(0)-2 m t)$ into $\left(\mathbb{R}^{n},\langle\cdot, \cdot\rangle\right)$ and the immersion $G: M \rightarrow \mathcal{H}^{n-1}(k)$ defined 
as $G:=I^{-1} \circ F$, as in the diagram

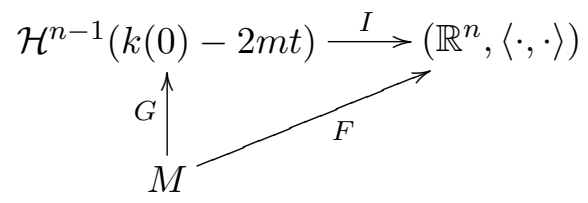

Writing $\vec{H}_{F}, \vec{H}_{G} \vec{H}_{I}$ for the respective mean curvature vectors, it holds:

- $\vec{H}_{F} \in T M^{\perp}$.

- $d I\left(\vec{H}_{G}\right) \in\left(d I\left(T \mathcal{H}^{n-1}\right)\right)$.

- $g^{i j}(\nabla d I)\left(d G\left(\frac{\partial}{\partial x^{i}}\right), d G\left(\frac{\partial}{\partial x^{j}}\right)\right) \in T \mathcal{H}^{n-1} \perp$.

But Equation (3.3) implies that $\vec{H}_{F} \in\left(T \mathcal{H}^{n-1}\right)^{\perp}$. Thus, $d I\left(\vec{H}_{G}\right)$ is the only term tangential to the hyperquadric in Equation (3.4), thence $d I\left(\vec{H}_{G}\right)=0$ and $\vec{H}_{G}=0$ (for $I$ is an immersion).

Further, we can calculate $-\frac{\dot{c}}{c}$. Let $t \in[0, T)$ be fixed and $x \in M$ be any point

$$
-\frac{\dot{c}}{c}\|F(x, t)\|^{2}=\left\langle\vec{H}_{F}, F\right\rangle=-\left\langle F_{i}, F_{j}\right\rangle g^{i j}=-m \Rightarrow-\frac{\dot{c}}{c}=-\frac{m}{\|F(t)\|^{2}}
$$

and Lemma 3.3 implies that

$$
\vec{H}_{F}(t)=-\frac{m}{\|F(0)\|^{2}-2 m t} F(t) .
$$

\subsection{Existence and uniqueness}

3.2.1. Immersion in the hyperquadric $\mathcal{H}^{\boldsymbol{n}-1}(\boldsymbol{k})$ with $\boldsymbol{k}>\mathbf{0}$. Let $\|F(x, 0)\|^{2}=k>0$ for all $x \in M$. Then $c(t)$ is given, from Equations (3.3) and $(3.5)$, by $c(t):=\sqrt{k}(k-2 m t)^{-1 / 2}$.

It follows from Equation (3.5), for any $(x, t) \in M \times[0, T)$, that (3.6)

$$
\frac{d}{d t} F(x, t)=\vec{H}_{F(\cdot, t)}(x)=-\frac{m}{k-2 m t} F(x, t)=-\frac{\dot{c}}{c} F(x, t) \Longrightarrow \frac{d}{d t}(c F(t))=0 .
$$

Hence $c F(x, t)=F(x, 0)$ and

$$
F(x, t)=\frac{1}{c} F(x, 0) .
$$


By construction, we proved that if there is a hyperquadric homothety of the MCF, then it has to be given by Equation (3.7). So the solution is unique in the class of hyperquadric homothetic solutions. We still have to deal with the question of existence. As in Theorem 3.4, a hyperquadric homothety of the MCF has to be a minimal surface of the hyperquadric. This motivates the following theorem:

Theorem 3.5. Let $F: M^{m} \rightarrow\left(\mathbb{R}^{n},\langle\cdot, \cdot\rangle\right)$ be an immersion such that $g:=$ $F^{*}\langle\cdot, \cdot\rangle$ is non-degenerate and $\|F\|^{2}=k \in \mathbb{R}, k>0$, then the solution of the $M C F$ of this initial immersion is a homothety if, and only if, $F: M \rightarrow$ $\mathcal{H}^{n-1}(k)$ is a minimal immersion in the hyperquadric $\mathcal{H}^{n-1}(k)$. The $M C F$ of $F$ has a solution $F: M \times[0, T) \rightarrow\left(\mathbb{R}^{n},\langle\cdot, \cdot\rangle\right)$ with $T=\frac{k}{2 m}$; moreover, the solution is $F(x, t):=c^{-1}(t) F(x)$, with $c(t):=\sqrt{k}(k-2 m t)^{-1 / 2}, \forall(x, t) \in$ $M \times[0, T)$.

Proof. We have to prove that the homothety given by Equation (3.7) is a solution of the MCF. Let us write $F(t):=F(\cdot, t)$ and $I$ for the inclusion of $\mathcal{H}^{n-1}(k)$ into $\left(\mathbb{R}^{n},\langle\cdot, \cdot\rangle\right)$ and $G:=I^{-1} \circ F$. By Equation (3.4) it follows:

$$
\vec{H}_{F(0)}=g^{i j}(\nabla d I)\left(d G\left(\frac{\partial}{\partial x^{i}}\right), d G\left(\frac{\partial}{\partial x^{j}}\right)\right)
$$

because $F(0)$ is a minimal immersion on the hyperquadric. Moreover, $\vec{H}_{F(0)}$ is orthogonal to $\mathcal{H}^{n-1}\left(\|F(0)\|^{2}\right.$ ), but so is $F(0)$, such that there is a function $\varphi: M \rightarrow \mathbb{R}$ with $\vec{H}_{F(0)}=\varphi F(0)$. One can calculate $\varphi$

$$
\begin{aligned}
\varphi\|F(0)\|^{2} & =\left\langle\vec{H}_{F(0)}, F(0)\right\rangle=-g^{i j}\left\langle\nabla_{j} F(0), \nabla_{i} F(0)\right\rangle=-m \\
& \Longrightarrow \vec{H}_{F(0)}=-\frac{m}{\|F(0)\|^{2}} F(0) .
\end{aligned}
$$

Now consider $F(t)=c^{-1}(t) F(0)$ (as in Equation (3.7)). Then $g^{i j}(t)=c^{2}(t)$ $g^{i j}(0)$ and

$$
\vec{H}_{F(t)}=c(t) \vec{H}_{F(0)}=-\frac{m}{\|F(0)\|^{2}} c(t) F(0) .
$$

On the other hand, for the function $c=\sqrt{k}(k-2 m t)^{-1 / 2}$

$$
\frac{d F(t)}{d t}=\frac{d}{d t}\left(\frac{1}{c(t)}\right) F(0)=-\frac{m}{\|F(0)\|^{2}} c(t) F(0)=\vec{H}_{F(t)} .
$$

Therefore this is a solution of the MCF. 


\subsubsection{Immersion in the hyperquadric $\mathcal{H}^{n-1}(k)$ with $k<0$.}

Theorem 3.6. Let $F: M^{m} \rightarrow\left(\mathbb{R}^{n},\langle\cdot, \cdot\rangle\right)$ be an immersion such that $g:=$ $F^{*}\langle\cdot, \cdot\rangle$ is non-degenerate and $\|F\|^{2}=k \in \mathbb{R}, k<0$, then the solution of the MCF of this initial immersion is a homothety if, and only if, $F: M \rightarrow$ $\mathcal{H}^{n-1}(k)$ is a minimal immersion in the hyperquadric $\mathcal{H}^{n-1}(k)$. The $M C F$ of $F$ has a solution $F(t): M \times[0, \infty) \rightarrow\left(\mathbb{R}^{n},\langle\cdot, \cdot\rangle\right)$; moreover, the solution is $F(x, t):=c^{-1}(t) F(x)$, with $c(t):=\sqrt{-k}(-k+2 m t)^{-1 / 2}$, for all $(x, t) \in$ $M \times[0, \infty)$.

Proof. Analogous to the previous subsection.

3.2.3. Immersion in the hyperquadric $\mathcal{H}^{\boldsymbol{n}-\mathbf{1}}(\mathbf{0})$. Let $F: M \times[0, T)$ be a homothety generated by the MCF with $\|F(x, 0)\|^{2}=0$ for all $x \in M$. From Lemma 3.3, it holds $\|F(x, t)\|^{2}=-2 m t$ if $F^{*}\langle\cdot, \cdot\rangle$ is non-degenerate, so that

$$
\|F(x, t)\|^{2}<0
$$

for all $(x, t) \in M \times(0, T)$.

On the other hand, $c(t) F(x, t) \in F(M, 0)$ because $F$ is a homothety, so that

$$
0=\|c(t) F(x, t)\|^{2}=c(t)^{2}\|F(x, t)\|^{2} .
$$

But $c(t) \neq 0$ because $F(M, t)=\{0\}$ for $c(t)=0$, which cannot be an immersion, then $\|F(x, t)\|^{2}=0$ for all $t \in[0, T)$. Which is a contradiction to Equation (3.8). So we proved

Theorem 3.7. There are no hyperquadric homotheties of the $M C F F$ : $M \times[0, T) \rightarrow\left(\mathbb{R}^{n},\langle\cdot, \cdot\rangle\right)$ with non-degenerate metric such that $F(M, 0) \subset$ $\mathcal{H}^{n-1}(0)$.

Remark 3.8. One could expect to find at least some stationary solutions in the light cone, like straight lines, but for such a line the metric is degenerate and thence this case is not included in Theorem 3.7.

Remark 3.9. But, as Ecker noted in [14], the upper light cone would immediately change to a hyperquadric and the explicitly solution to the MCF with the upper light cone as initial condition in $\mathbb{R}^{1, n}$ is given by the 
family of graphs $\delta_{t}: \mathbb{R}^{n-1} \rightarrow \mathbb{R}$

$$
\delta_{t}(x)=\sqrt{\|x\|_{\mathbb{E}}^{2}+2(n-1) t}
$$

for any $t \in[0, \infty)$, which is a homothety after $t=0$.

Remark 3.10. If $k>0$ then $F$ and $\vec{H}$ are pointing in opposite directions and $F_{0}(M)$ shrinks under the MCF.

If $k<0$ then $F$ and $\vec{H}$ are pointing in the same direction and $F_{0}(M)$ expands under the MCF.

The principal normal is the vector field

$$
\nu:=\frac{\vec{H}}{\sqrt{\left|\|\vec{H}\|^{2}\right|}} .
$$

Remark 3.11. It is clear from Equation (3.5) that $\vec{H} \neq 0$ everywhere for a hyperquadric homothety of the MCF and $\nabla^{\perp} \vec{H}=\nabla^{\perp} \nu=0$.

\section{Principal normal parallel in the normal bundle}

Several calculations are carried out in this section in order to obtain an equation for $\triangle\|P\|^{2} /\|\vec{H}\|^{4}$ suitable for the maximum principle.

The two types of homotheties (self-shrinkers and self-expanders) lead, after rescaling, to different equations $\vec{H}=-F^{\perp}$ or $\vec{H}=F^{\perp}$. We restrict our attention, in this section, to the self-shrinkers of the MCF that have the principal normal parallel in the normal bundle.

If one considers a complexification of the tangent and normal bundles, $\frac{\vec{H}}{\|\vec{H}\| \mid}$ parallel in the normal bundle is equivalent ${ }^{5}$ to the possibly imaginary vector field $\nu:=\frac{\vec{H}}{\|\vec{H}\|}$ being parallel in the normal bundle.

We prove in this section that a compact spacelike self-shrinker cannot satisfy $\|\vec{H}\|^{2}<m$ for all $x \in M$. And we generalize Smoczyk's classification to the pseudo-Euclidean spaces

Theorem 4.1. Let $M$ be a closed smooth manifold and $F: M \rightarrow\left(\mathbb{R}^{n},\langle\cdot, \cdot\rangle\right)$ be a smooth immersion, which is a spacelike self-shrinker of the $M C F$, i.e.,

\footnotetext{
${ }^{5}$ Assuming $\|H\|^{2} \neq 0 \forall x \in M$.
} 
$F$ satisfies

$$
\vec{H}=-F^{\perp} .
$$

Besides, assume $m:=\operatorname{dim}(M) \neq 1$. Then the mean curvature vector $\vec{H}$ satisfies $\|\vec{H}\|^{2}(p) \neq 0$ for all $p \in M$ and the principal normal $\nu$ is parallel in the normal bundle $\left(\nabla^{\perp} \nu \equiv 0\right)$ if, and only if, $F$ is a minimal immersion in the hyperquadric $\mathcal{H}^{n-1}(m)$.

\subsection{Fundamental equations}

This subsection closely follows Smoczyk's calculations, which we refer to for details. We make use of three auxiliary tensors

$$
P_{i j}:=\left\langle\vec{H}, A_{i j}\right\rangle, \quad Q_{i j}:=\left\langle A_{i}^{k}, A_{k j}\right\rangle, \quad S_{i j k l}:=\left\langle A_{i j}, A_{k l}\right\rangle .
$$

Using Gauß equation (Equation (2.2)), the Ricci curvature is written as

$$
R_{i j}=g^{k l} R_{k i l j}=g^{k l}\left\langle A_{l k}, A_{j i}\right\rangle-g^{k l}\left\langle A_{j k}, A_{l i}\right\rangle=P_{i j}-Q_{i j} .
$$

In this notation, the useful Simons' equation is written as

$$
\nabla_{k}^{\perp} \nabla_{l}^{\perp} \vec{H}=\triangle^{\perp} A_{k l}+R_{k i l j} A^{i j}-R_{k}^{i} A_{i l}+Q_{l}^{i} A_{i k}-S_{k i l j} A^{i j} .
$$

If we fix $t \in[0, T)$ the immersion $F_{t}$ can be constant rescaled to bring Equation (3.3) into

$$
\vec{H}=-F^{\perp} .
$$

On the other hand, in ([19]) Huisken shows that an isometric immersion satisfying $\vec{H}=-F^{\perp}$ is (up to a tangential component) the MCF.

We make use of the following one-form $\theta$ :

$$
\theta:=\frac{1}{2} d\|F\|^{2}=\left\langle F_{i}, F\right\rangle d x^{i}
$$

such that $\theta^{j} F_{j}=\theta_{i} g^{i j} F_{j}$ is equal to $F^{\top}$, with $\theta_{i}=\left\langle F_{i}, F\right\rangle$. Then

$$
\nabla_{i} \theta_{j}=\nabla_{i}\left\langle F_{j}, F\right\rangle=\left\langle A_{i j}, F\right\rangle+g_{i j}
$$

Hence it follows

$$
\nabla_{i}^{\perp} F^{\perp}=\left(\nabla_{i}\left(F-\theta^{k} F_{k}\right)\right)^{\perp}=\left(F_{i}-\nabla_{i} \theta^{k} F_{k}-\theta^{k} A_{i k}\right)^{\perp}=-\theta^{k} A_{i k}
$$


and

$$
\nabla_{i}^{\perp} \vec{H}=-\nabla_{i}^{\perp} F^{\perp}=\theta^{k} A_{i k}
$$

So that

$$
\nabla_{i}^{\perp} \nabla_{j}^{\perp} F^{\perp}==-A_{i j}-\left\langle A_{i}^{k}, F^{\perp}\right\rangle A_{j k}-\theta^{k} \nabla_{k}^{\perp} A_{i j}
$$

where we used the Codazzi equation (Theorem 2.1) in the last step. From this follows that

$$
\nabla_{i}^{\perp} \nabla_{j}^{\perp} \vec{H}=-\nabla_{i}^{\perp} \nabla_{j}^{\perp} F^{\perp}=A_{i j}-P_{i}^{k} A_{k j}+\theta^{k} \nabla_{i}^{\perp} A_{j k}
$$

and

$$
\triangle^{\perp} \vec{H}=g^{i j} \nabla_{i}^{\perp} \nabla_{j}^{\perp} \vec{H}=\vec{H}-P^{i k} A_{i k}+\theta^{k} \nabla_{k}^{\perp} \vec{H}
$$

Hence

$$
\triangle\|\vec{H}\|^{2}=2\|\vec{H}\|^{2}-2\|P\|^{2}+2\left\|\nabla^{\perp} \vec{H}\right\|^{2}+\left\langle F^{\top}, \nabla\|\vec{H}\|^{2}\right\rangle .
$$

For $\|A\|^{2}$, using Simons' equation (Proposition 4.3), one gets

$$
\begin{aligned}
2\left\langle A,\left(\nabla^{\perp}\right)^{2} \vec{H}\right\rangle= & g^{t k} g^{s l} 2\left\langle A_{t s}, \triangle^{\perp} A_{k l}+R_{k i l j} A^{i j}-R_{k}^{i} A_{i l}+Q_{l}^{i} A_{i k}-S_{k i l j} A^{i j}\right\rangle \\
= & \triangle\|A\|^{2}-2\left\|\nabla^{\perp} A\right\|^{2}+2 R_{k i l j} S^{i j k l}-2 R_{i j} Q^{i j}+2\|Q\|^{2} \\
& -2 S_{i k j l} S^{i j k l} .
\end{aligned}
$$
holds

But, using Equation (2.4) for the Ricci tensor of the normal bundle, it

$$
\left\|R^{\perp}\right\|^{2}=Q_{k l} Q^{k l}-S_{i k j l} S^{k j l i}-S_{j k i l} S^{k i l j}+Q_{k l} Q^{k l}=2\|Q\|^{2}-2 S_{i k j l} S^{i j k l} .
$$

So that, using these last two equations, we reach

$$
2\left\langle A,\left(\nabla^{\perp}\right)^{2} \vec{H}\right\rangle=\triangle\|A\|^{2}-2\left\|\nabla^{\perp} A\right\|^{2}+2\|S\|^{2}-2\langle P, Q\rangle+2\left\|R^{\perp}\right\|^{2} .
$$

On the other hand, we can calculate an equation for $\triangle\|A\|^{2}$ using Simons' equation (Proposition 4.3) in the following way: first, with Equations (4.7) and (4.2), we have

$$
\begin{aligned}
& \triangle^{\perp} A_{k l}=\nabla_{k}^{\perp} \nabla_{l}^{\perp} \vec{H}-R_{k i l j} A^{i j}+R_{k}^{i} A_{i l}-Q_{l}^{i} A_{i k}+S_{k i l j} A^{i j}, \\
& \triangle^{\perp} A_{k l}=A_{k l}-Q_{k}^{i} A_{i l}-Q_{l}^{i} A_{i k}+\theta^{t} \nabla_{k}^{\perp} A_{l t}+\left(S_{k i l j}-R_{k i l j}\right) A^{i j}
\end{aligned}
$$


which implies

$$
\triangle\|A\|^{2}=2\|A\|^{2}-2\left\|R^{\perp}\right\|^{2}+\left\langle F^{\top}, \nabla\|A\|^{2}\right\rangle-2\|S\|^{2}+2\left\|\nabla^{\perp} A\right\|^{2} .
$$

Theorem 4.2. Let $M$ be a closed smooth manifold and $F: M \rightarrow\left(\mathbb{R}^{n},\langle\cdot, \cdot\rangle\right)$ be a spacelike self-shrinker of the $M C F$. Then it cannot hold $\|\vec{H}\|^{2}<m:=$ $\operatorname{dim}(M)$.

Proof. If $\|\vec{H}\|^{2}<m$ for all $x \in M$, then

$$
\triangle\|F\|^{2}=2 g^{i j}\left\langle F_{i}, F_{j}\right\rangle+2\langle\triangle F, F\rangle=2 m-2\|\vec{H}\|^{2}>0 .
$$

But at a maximum $p$ of $\|F\|^{2}$ it holds $\triangle\|F\|^{2} \leq 0$. Which is a contradiction.

Remark 4.3. In particular, there are no spacelike self-shrinkers with $\|\vec{H}\|^{2}<0$ and no spacelike self-shrinkers if the index of $\left(\mathbb{R}^{n},\langle\cdot, \cdot\rangle\right)$ is $n-m$.

\subsection{The compact case}

Let us now consider the self-shrinkers of the MCF that satisfy the following conditions:

- The mean curvature vector $\|\vec{H}(x)\|^{2} \neq 0$, for all $x \in M$.

- The principal normal $\nu:=\frac{1}{\|\vec{H}\|} \vec{H}$ is parallel in the normal bundle $\nabla^{\perp} \nu \equiv 0$

where we write $\|\vec{H}\|$ to the complex function $\sqrt{\|\vec{H}\|^{2}}: M \rightarrow \mathbb{C}$. Although Theorem 4.2 implies that $\|\vec{H}\|^{2} \geq 0$ in the compact case, we also consider $\|\vec{H}\|^{2} \leq 0$ as a possibility for the calculations bellow for they are of use in the non-compact case.

The complex function $\|\vec{H}\|$ is a pure real or a pure imaginary all over $M$. So $\nu$ may not to be a real vector, but a vector field in the complexification of the pullback over $M$ of $T \mathbb{R}^{n}$. Over this bundle we extend the inner product and the connection linearly. Additionally for $X \in \Gamma\left(F^{*} T \mathbb{R}^{n}\right)$, we use $(i X)^{\perp}:=i\left(X^{\perp}\right)$.

Remark 4.4. The equations considered bellow are real or pure imaginary. Thence there will be not explicit mentions of the complexifications in the calculations. 
A parallel principal normal (in the normal bundle) can simplify some of the previously calculated equations because of its properties

$$
\nabla_{k}^{\perp} \vec{H}=\nabla_{k}^{\perp}(\|\vec{H}\| \nu)=\nabla_{k}\|\vec{H}\| \nu
$$

and

$$
\triangle^{\perp} \vec{H}=g^{i j} \nabla_{i}^{\perp} \nabla_{j}^{\perp}(\|\vec{H}\| \nu)=g^{i j} \nabla_{i} \nabla_{j}\|\vec{H}\| \nu=\triangle\|\vec{H}\| \nu .
$$

From this, using Equation (4.8), we calculate

$$
P^{i j} A_{i j}=\vec{H}+\theta^{k} \nabla_{k}^{\perp} \vec{H}-\triangle^{\perp} \vec{H}=\left(\|\vec{H}\|+\theta^{k} \nabla_{k}\|\vec{H}\|-\triangle\|\vec{H}\|\right) \nu
$$

which means that $P^{i j} A_{i j}$ is in the same direction as $\nu$ (or $i \nu$, if $\nu$ is imaginary).

Lemma 4.5. Let $F: M \rightarrow\left(\mathbb{R}^{n},\langle\cdot, \cdot\rangle\right)$ be an immersion such that the principal normal is parallel in normal bundle, then

$$
\begin{array}{lll}
\text { (1) } \quad P^{i j} A_{i j}=\frac{\|P\|^{2}}{\|\vec{H}\|} \nu, & \text { (2) } & S_{i j k l} P^{i j} P^{k l}=\frac{\|P\|^{4}}{\|\vec{H}\|^{2}} \\
\text { (3) } \quad P_{i}^{k} A_{k j}=P_{j}^{k} A_{k i}, & \text { (4) } \quad S_{i k j l} P^{i j} P^{k l}=Q_{i l} P_{k}^{i} P^{k l} .
\end{array}
$$

Lemma 4.6. Let $F: M \rightarrow\left(\mathbb{R}^{n},\langle\cdot, \cdot\rangle\right)$ be a self-shrinker of the $M C F$ such that the principal normal is parallel in normal bundle, then

$$
\frac{4}{\|\vec{H}\|^{4}}\left\langle\nabla^{\perp} \vec{H}, \nabla^{\perp} A_{i j}\right\rangle P^{i j}=\frac{2}{\|\vec{H}\|}\left\langle\nabla\|\vec{H}\|, \nabla\left(\frac{\|P\|^{2}}{\|\vec{H}\|^{4}}\right)\right\rangle+4 \frac{\|P\|^{2}}{\|\vec{H}\|^{6}}\|\nabla\| \vec{H}\|\|^{2} .
$$

Lemma 4.7. Let $F: M \times[0, T) \rightarrow\left(\mathbb{R}^{n},\langle\cdot, \cdot\rangle\right)$ be a self-shrinker of the $M C F$ such that $\|\vec{H}\|^{2} \neq 0$ for all $x \in M$ and the principal normal is parallel in the normal bundle. Then

$$
\begin{aligned}
\triangle\left(\frac{\|P\|^{2}}{\|\vec{H}\|^{4}}\right)= & \frac{2}{\|\vec{H}\|^{4}}\left\|\nabla_{i}\right\| \vec{H}\left\|\frac{P_{j k}}{\|\vec{H}\|}-\right\| \vec{H}\left\|\nabla_{i}\left(\frac{P_{j k}}{\|\vec{H}\|}\right)\right\|^{2} \\
& +\left\langle F^{\top}, \nabla\left(\frac{\|P\|^{2}}{\|\vec{H}\|^{4}}\right)\right\rangle-\frac{2}{\|\vec{H}\|}\left\langle\nabla\|\vec{H}\|, \nabla\left(\frac{\|P\|^{2}}{\|\vec{H}\|^{4}}\right)\right\rangle .
\end{aligned}
$$

Proposition 4.8. Let $M$ be a closed smooth manifold and $F: M \rightarrow$ $\left(\mathbb{R}^{n},\langle\cdot, \cdot\rangle\right)$ be a smooth immersion, which is a spacelike self-shrinker of the 
MCF. Besides, assume that the mean curvature vector $\vec{H}$ satisfies $\|\vec{H}\|^{2} \neq 0$ and the principal normal $\nu$ satisfies $\nabla^{\perp} \nu=0$. Then

$$
\left\|\nabla_{i}\right\| \vec{H}\left\|\frac{P_{j k}}{\|\vec{H}\|}-\right\| \vec{H}\left\|\nabla_{i}\left(\frac{P_{j k}}{\|\vec{H}\|}\right)\right\|^{2}=0 .
$$

Proof. Although the function $\|\vec{H}\|$ may be imaginary, the 3-tensor in Equation (4.20) is real. Thus the strong elliptic maximum principle implies that $\|P\|^{2} /\|\vec{H}\|^{4}$ is constant. Hence theorem 4.7 implies that

$$
\left\|\nabla_{i}\right\| \vec{H}\left\|\frac{P_{j k}}{\|\vec{H}\|}-\right\| \vec{H}\left\|\nabla_{i}\left(\frac{P_{j k}}{\|\vec{H}\|}\right)\right\|^{2}=0 .
$$

The equality we just proved can be written as

$$
\|\nabla\| \vec{H}\|\|^{2}\|P\|^{2}-\left\|\nabla_{i}\right\| \vec{H}\left\|P_{k}^{i}\right\|^{2}=0 .
$$

The rest of the proof is important also in the non-compact case. We follow in detail.

What remains to prove of Theorem 4.1. Let $M$ be a closed smooth manifold and $F: M \rightarrow\left(\mathbb{R}^{n},\langle\cdot, \cdot\rangle\right)$ be an immersion, which is a spacelike selfshrinker of the MCF. Besides, assume that the mean curvature vector $\vec{H}$ satisfies $\|\vec{H}\|^{2} \neq 0$ and the principal normal $\nu$ satisfies $\nabla^{\perp} \nu=0$. If $m:=$ $\operatorname{dim}(M) \neq 1$, then

$$
\|F(x)\|^{2}=m, \forall x \in M .
$$

Proof. We now calculate at a point $p \in M$ fixed. As the 2-tensor $P$ is symmetric, it is also diagonalizable and has only real eigenvalues $\lambda_{1}, \ldots, \lambda_{m}$. Let $V_{1}, \ldots, V_{m}$ be an orthonormal basis of eigenvectors associated with $\lambda_{1}, \ldots, \lambda_{m}$. Then we write $\nabla\|\vec{H}\|=\sum_{i} \alpha_{i} V_{i}, \quad \alpha_{i} \in \mathbb{C}$ so that by Equation (4.21)

$$
0=\|P\|^{2}\|\nabla\| \vec{H}\|\|^{2}-\|P(\nabla\|\vec{H}\|)\|^{2}=\sum_{i} \lambda_{i}^{2}\left(\|\nabla\| \vec{H}\|\|^{2}-\alpha_{i}^{2}\right)
$$

but $\lambda_{i}^{2} \geq 0$, because $\lambda_{i} \in \mathbb{R}$, beyond this

$$
\|\nabla\| \vec{H}\|\|^{2}-\alpha_{i}^{2}=\sum_{j \neq i} \alpha_{j}^{2}
$$


is non-negative for all $i \in\{1, \ldots, m\}$ if $\|\vec{H}\|$ is real or non-positive if $\|\vec{H}\|$ is imaginary. This implies, with Equation (4.22), that

$$
\lambda_{i}^{2}\left(\|\nabla\| \vec{H}\|\|^{2}-\alpha_{i}^{2}\right)=0 \quad \forall i \in\{1, \ldots, m\} .
$$

As $\operatorname{tr}(P)=P_{i j} g^{i j}=\|\vec{H}\|^{2} \neq 0$, it follows that $P \neq 0$ and there is at least one $j \in\{1, \ldots, n\}$ such that $\lambda_{j} \neq 0$ and the last equation shows that

$$
0=\|\nabla\| \vec{H}\|\|^{2}-\alpha_{j}^{2}=\sum_{i} \alpha_{i}^{2}-\alpha_{j}^{2}=\sum_{i \neq j} \alpha_{i}^{2} \Longrightarrow \alpha_{i}=0 \forall i \neq j
$$

because the $\alpha_{i}$ 's are all real or all imaginary. From this follows that $\nabla\|\vec{H}\|=$ $\alpha_{j} V_{j}$.

Assume that there is an $x \in M$ such that $\nabla\|\vec{H}\|(x) \neq 0$.

Then $\alpha_{j} \neq 0$ and for all $i \neq j$

$$
0=\lambda_{i}^{2}\left(\|\nabla\| \vec{H}\|\|^{2}-\alpha_{i}^{2}\right)=\lambda_{i}^{2} \alpha_{j}^{2} \Longrightarrow \lambda_{i}=0
$$

so $P_{i j}$ has only one non-zero eigenvalue and the associated eigenvector is $\nabla\|\vec{H}\| /\|\nabla\| \vec{H}\|\|$.

At this point, we have

$$
\|P\|^{2}=\lambda_{j}^{2}=(\operatorname{tr} P)^{2}=\|\vec{H}\|^{4} \Longrightarrow \frac{\|P\|^{2}}{\|\vec{H}\|^{4}}=1
$$

but $\|P\|^{2} /\|\vec{H}\|^{4}$ is constant, thence equal 1 everywhere in $M$.

Then, using $\|P\|^{2}=\|\vec{H}\|^{4}$, with Equation (4.9) we calculate

$$
2\|\vec{H}\| \triangle\|\vec{H}\|=2\|\vec{H}\|^{2}-2\|\vec{H}\|^{4}+2\|\vec{H}\|\left\langle F^{\top}, \nabla\|\vec{H}\|\right\rangle
$$

and it follows

$$
\triangle\|\vec{H}\|=\|\vec{H}\|-\|\vec{H}\|^{3}+\left\langle F^{\top}, \nabla\|\vec{H}\|\right\rangle
$$

We integrate both sides of this equation to get

$$
0=\int_{M} \triangle\|\vec{H}\|=\int_{M}\|\vec{H}\|-\|\vec{H}\|^{3}+\left\langle F^{\perp}, \nabla|\vec{H}|\right\rangle=(1-m) \int_{M}\|\vec{H}\|
$$

which is impossible for $m \neq 1$.

From this contradiction, we know that $\nabla\|\vec{H}\|=0$ everywhere in $M$. It follows that $\nabla^{\perp} \vec{H}=\nabla\|\vec{H}\| \nu=0$ and that the norm of $\vec{H}$ is constant. 
On the other hand

$$
\triangle\|F\|^{2}=2\left\langle F_{i}, F_{j}\right\rangle g^{i j}+2\langle\triangle F, F\rangle=2 g_{i j} g^{i j}+2\langle\vec{H}, F\rangle=2 m-2\|H\|^{2} .
$$

By the maximum principle $\|F\|^{2}$ is constant. This norm can be calculated seeing that $\left\langle F_{i}, F\right\rangle=0$, which implies that $F \in \Gamma\left(T M^{\perp}\right)$, so that $\vec{H}=-F$ and replacing $\|F\|^{2}=\|\vec{H}\|^{2}$ and $\triangle\|F\|^{2}=0$ in Equation (4.24) we get $\|F\|^{2}=\|\vec{H}\|^{2}=m$.

Note that the condition $\operatorname{dim}(M) \neq 1$ is optimal, because the result does not hold for the curve shortening flow, then the Abresch \& Langer curves are not contained in a circle.

\section{The non-compact case}

Now non-compact self-shrinkers are considered. We need to establish Equation (4.20) also in this case, for that we use some integrals over the whole $M$ with respect to some heat kernel. This section starts with the lemmas and definitions needed to guarantee the convergence of these integrals. In particular, $F(M)$ has to stay away from the light cone in the sense of Definition 5.4, which, of course, does not come for in the Euclidean case. Then we follow as in the compact case up to Equation (4.23).

Remark 5.1. In the pseudo-Euclidean case, there are minimal submanifolds of the hyperquadrics, which are non-compact and are homotheties of the MCF with principal normal parallel in the normal bundle. These hyperquadrics are asymptotic to the light cone and, in particular, have the norm $\|F\|^{2}$ bounded, thence they do not satisfy the conditions needed to integrate and do not appear in our results.

In similar fashion to the compact case it holds:

Theorem 5.2. The mean curvature vector of a stochastic complete, spacelike, self-shrinker of the $M C F F: M \rightarrow\left(\mathbb{R}^{n},\langle\cdot, \cdot\rangle\right)$ cannot satisfy, for all $p \in M$

$$
\|\vec{H}\|^{2}<m-\epsilon
$$

for some $\epsilon>0$ if $\sup _{M}\|F\|^{2}<+\infty$. 
Proof. If there is an $\epsilon>0$ such that $\|\vec{H}\|^{2}<m-\epsilon$ for all $x \in M$, then

$$
\triangle\|F\|^{2}=2 g^{i j}\left\langle F_{i}, F_{j}\right\rangle+2\langle\triangle F, F\rangle=2 m-2\|\vec{H}\|^{2}>2 \epsilon
$$

which leads to a contradiction by the weak Omori-Yau maximum principle.

Remark 5.3. In particular, there are no stochastic complete, spacelike self-shrinkers of the MCF with $\sup _{M}\|F\|^{2}<+\infty$ and $\|\vec{H}\|^{2} \leq 0$.

Let $\left(\mathbb{R}^{n},\langle\cdot, \cdot\rangle\right)$ be an inner product space and $\left\{e_{1}, \ldots, e_{n}\right\}$ an orthonormal basis such that $\left\langle e_{\alpha}, e_{\alpha}\right\rangle=-1$ for $\alpha \in\{1, \ldots, q\}$ and $\left\langle e_{\alpha}, e_{\alpha}\right\rangle=1$ for $\alpha \in\{q+$ $1, \ldots, n\}$, which we denote $\mathbb{R}^{q, n}$. We write $\left(X_{-}\right)$and $\left(X_{+}\right)$for the projections of $X$ on $\operatorname{span}\left\{e_{1}, \ldots, e_{q}\right\}$ and $\operatorname{span}\left\{e_{q+1}, \ldots, e_{n}\right\}$, respectively.

Definition 5.4. Let $M$ be a smooth manifold and $F: M \rightarrow \mathbb{R}^{q, n}$ be an immersion with $F(M)$ unbounded. We say that $F$ (or $F(M)$ ) is mainly positive if there is an $\epsilon>0$ and $k \in \mathbb{R}$, such that $\forall x \in M$

$$
\|F(x)\|_{\mathbb{E}}^{2} \geq k \Longrightarrow-\frac{\left\|F(x)_{-}\right\|^{2}}{\left\|F(x)_{+}\right\|^{2}} \leq 1-\epsilon .
$$

And we say that $F$ (or $F(M)$ ) is mainly negative if there is an $\epsilon>0$ and $k \in \mathbb{R}$, such that $\forall x \in M:\|F(x)\|_{\mathbb{E}}^{2} \geq k \Longrightarrow-\frac{\left\|F(x)_{+}\right\|^{2}}{\left\|F(x)_{-}\right\|^{2}} \leq 1-\epsilon$.

This definition is illustrated bellow for a plane curve:

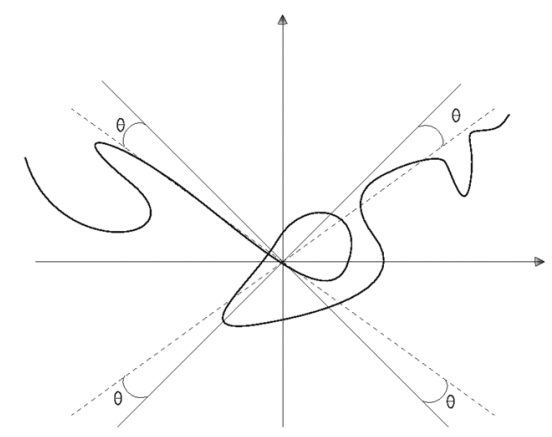

Mainly positive means that there is an (Euclidean) angle $\theta$ with tan $\left(\frac{\pi}{4}-\theta\right)<1-\epsilon$ between $F(x)$ and the light cone for any $x \in M$ such that $F(x)$ lies outside some big Euclidean sphere (or $\tan \left(\frac{\pi}{4}-\theta\right)>1+\epsilon$ in the mainly negative case).

In both of the cases $\|F\|^{2}$ is unbounded. Thence follows: 
Remark 5.5. If $F$ is a spacelike self-shrinker such that $F(M)$ is mainly negative and unbounded, then for $x \in M$ with $\|F(x)\|_{\mathbb{E}}^{2}>k$, for $k$ as in Definition 5.4, it holds that

$$
0>\|F(x)\|^{2}=\left\|F^{\perp}(x)\right\|^{2}+\left\|F^{\top}(x)\right\|^{2} \geq\|\vec{H}(x)\|^{2}
$$

but if $M$ is stochastic complete, then Theorem 5.2 implies that $F$ cannot be a self-shrinker of the MCF with $\|\vec{H}\|^{2}(p) \neq 0$ for all $p \in M$.

In order to integrate, we need further assumptions on $F$ :

Definition 5.6. Let $F: M \rightarrow \mathbb{R}^{q, n}$ be a spacelike isometric immersion. We say that $F$ has bounded geometry if:

(1) There are $c_{k}, d_{k} \in \mathbb{R}, \quad k \in \mathbb{N}$, such that $\left\|(\nabla)^{k} A_{+}\right\|^{2} \leq c_{k}$ and $-\left\|(\nabla)^{k} A_{-}\right\|^{2} \leq d_{k}$.

(2) The function $\frac{1}{\|\vec{H}\|}$ grows polynomially with respect to $\|F\|^{2}$.

(3) The growth of volume of geodesic balls and their boundaries is polynomial with respect to the radius.

(4) $F$ is inverse Lipschitz with respect to the Euclidean norm in $\mathbb{R}^{n}$.

Then the bounded geometry assumption excludes the mainly negative case for spacelike self-shrinkers, because $-d_{0} \leq\|\vec{H}\|^{2} \leq c_{0}$ and, in this case, $\|F\|^{2}$ has no lower bound, but $\|F\|^{2}=\left\|F^{\top}\right\|^{2}+\left\|F^{\perp}\right\|^{2}$ and $\left\|F^{\top}\right\|^{2} \geq 0$, which implies that $\left\|F^{\perp}\right\|^{2}$ is not bounded below. This contradicts $\left\|F^{\perp}\right\|^{2}=$ $\|\vec{H}\|^{2}$. So that:

Theorem 5.7. There are no unbounded mainly negative spacelike selfshrinkers of the MCF with bounded geometry.

We will integrate over the whole manifold with respect to the following heat kernel: $\rho: M \rightarrow \mathbb{R}$ defined as

$$
\rho(x):=\exp \left(-\frac{\|F\|^{2}}{2}\right) .
$$

Lemma 5.8. Let $F: M \rightarrow \mathbb{R}^{q, n}$ be a spacelike, mainly positive, immersion with bounded geometry and $F(M)$ unbounded, beyond this let $f: M \rightarrow \mathbb{R}$ be some polynomial (of inner products) of $\vec{H}, A$, their covariant derivatives, 
$F, F^{\top}$ and the function $\frac{1}{\|\vec{H}\|}$. Then

$$
\left|\int_{M} f \rho d \mu\right|<\infty
$$

beyond this, one can use partial integration

$$
\int_{M} \rho \operatorname{div}(\nabla f(x)) d \mu=-\int_{M}\langle\nabla \rho, \nabla f(x)\rangle d \mu .
$$

By this, all integrals in the next lemma are finite.

Lemma 5.9. Let $F: M \rightarrow \mathbb{R}^{q, n}$ be a spacelike, mainly positive, self-shrinker of the MCF with bounded geometry such that $F(M)$ is unbounded. Beyond this, let $F$ satisfy $\|\vec{H}\|^{2} \neq 0$ and $\nabla^{\perp} \nu=0$. Then

$$
\nabla_{i}\|\vec{H}\| \frac{P_{j k}}{\|\vec{H}\|}-\|\vec{H}\| \nabla_{i}\left(\frac{P_{j k}}{\|\vec{H}\|}\right)=0 .
$$

Proof. The expression

$$
\int_{M} \rho \frac{\|P\|^{2}}{\|\vec{H}\|^{2}} \triangle\left(\frac{\|P\|^{2}}{\|\vec{H}\|^{4}}\right) d \mu
$$

can be calculated using partial integration or Lemma 4.7. Equaling these two one finds

$$
\begin{gathered}
\int_{M} 2 \rho \frac{\|P\|^{2}}{\|\vec{H}\|^{6}}\left\|\nabla_{i}\right\| \vec{H}\left\|\frac{P_{j k}}{\|\vec{H}\|}-\right\| \vec{H}\left\|\nabla_{i}\left(\frac{P_{j k}}{\|\vec{H}\|}\right)\right\|^{2} \\
+\rho\|\vec{H}\|^{2}\left\|\nabla\left(\frac{\|P\|^{2}}{\|\vec{H}\|^{4}}\right)\right\|^{2} d \mu=0
\end{gathered}
$$

but the two summands inside the integral have the same sign everywhere. This implies in particular, using $\|P\|^{2} \neq 0$ (because $P=0$ would imply $\|\vec{H}\|=0)$, that

$$
\nabla_{i}\|\vec{H}\| \frac{P_{j k}}{\|\vec{H}\|}-\|\vec{H}\| \nabla_{i}\left(\frac{P_{j k}}{\|\vec{H}\|}\right)=0
$$

And we have the same result as Equation 4.20 in the compact case. Then we follow exactly as in the previous section (the compact case) to get: 
Lemma 5.10. Let $M$ be a smooth manifold and $F: M \rightarrow \mathbb{R}^{q, n}$ be mainly positive, spacelike, self-shrinker of the MCF with bounded geometry such that $F(M)$ is unbounded, beyond that let $F$ satisfy the conditions: $\|\vec{H}\|^{2}(p) \neq 0$ for all $p \in M$ and the principal normal is parallel in the normal bundle $\left(\nabla^{\perp} \nu \equiv 0\right)$. Then one of the two holds

(1) $\nabla\|\vec{H}\|=0$ everywhere on $M$.

(2) There is a point $p \in M$ with $\nabla\|\vec{H}\|(p) \neq 0$, at which $\frac{\nabla\|\vec{H}\|}{\|\nabla\| \vec{H}\|\|}$ is the only eigenvector associated with a non-zero eigenvalue of $P$.

We have to treat these two cases separately.

\section{The first case}

For the strategy of this section, see the last paragraph of the introduction.

Theorem 6.1. Let $M$ be a smooth manifold and $F: M \rightarrow \mathbb{R}^{q, n}$ be a mainly positive, spacelike, shrinking self-similar solution of the MCF with bounded geometry such that $F(M)$ is unbounded. Beyond that, let $F$ satisfy the conditions: $\|\vec{H}\|^{2}(p) \neq 0, \forall p \in M$, and the principal normal is parallel in the normal bundle $\left(\nabla^{\perp} \nu \equiv 0\right)$. If $\nabla\|\vec{H}\|(p)=0$ for all $p \in M$, then

$$
F(M)=\mathcal{H}_{r} \times \mathbb{R}^{m-r},
$$

where $\mathcal{H}_{r}$ is an $r$-dimensional minimal surface of the hyperquadric $\mathcal{H}^{n-1}(r)$ with $\|\vec{H}\|^{2}=r>0$ and $R^{m-r}$ is an $m-r$ dimensional spacelike affine space in $\mathbb{R}^{q, n}$.

Proof. First we see that $\nabla\|\vec{H}\|=0$ implies $\nabla^{\perp} \vec{H}=\nabla\|\vec{H}\| \nu=0$ and, with Equation (4.6)

$$
\theta^{i} A_{i j}=0
$$

On the other hand, $\nabla\|\vec{H}\|=0$ implies that $\|\vec{H}\|^{2}$ is constant, so that, with Lemma 5.9, it holds $\nabla P=0$ and then Equation (4.7) implies

$$
\begin{aligned}
\left\langle\nabla_{i}^{\perp} \nabla_{j}^{\perp} \vec{H}, \vec{H}\right\rangle & =\left\langle A_{i j}-P_{i}^{k} A_{k j}+\theta^{k} \nabla_{i}^{\perp} A_{j k}, \vec{H}\right\rangle, \\
0 & =P_{i j}-P_{i}^{k} P_{k j}
\end{aligned}
$$

so that $P=P^{2}$, i.e., $P$ is a projection and can only have 1 and 0 as eigenvalues. 
Because of $\nabla_{k} P_{i j}=0$ we get $\nabla_{k}\|P\|^{2}(p)=0$, but $\|P\|^{2}(p)$ is equal to the number of eigenvalues 1 , thus their number is constant and

$$
\|\vec{H}\|^{2}=\operatorname{tr} P=r>0 .
$$

We consider the eigenspaces associated with these two eigenvalues, they define the distributions $\mathcal{E} M$ and $\mathcal{F} M$ given, at any point $p \in M$, by

$$
\mathcal{E} M_{p}:=\left\{V \in T_{p} M: P_{i}^{j} V^{i}=V^{j}\right\}, \quad \mathcal{F} M_{p}:=\left\{V \in T_{p} M: P_{i}^{j} V^{i}=0\right\} .
$$

And $T_{p} M=\mathcal{E} M_{p} \oplus \mathcal{F} M_{p}$. From Equation (6.2) we have, for all $V \in \mathcal{E}_{p} M$, that

$$
\theta(V)=\theta_{j} V^{j}=\theta_{j} P_{i}^{j} V^{i}=0
$$

Lemma 6.2. Under the hypothesis of Theorem 6.1, the distributions $\mathcal{E} M$ and $\mathcal{F} M$ are involutive.

Proof. For $e_{1}, e_{2} \in \Gamma(\mathcal{E} M)$ and $f_{1}, f_{2} \in \Gamma(\mathcal{F} M)$, from $\nabla P=0$, we get

$$
\begin{aligned}
& P\left(\nabla_{e_{1}} e_{2}\right)=\nabla_{e_{1}} P\left(e_{2}\right)=\nabla_{e_{1} e_{2}}, \\
& P\left(\nabla_{f_{1}} f_{2}\right)=\nabla_{f_{1}} P\left(f_{2}\right)=\nabla_{f_{1}} 0=0
\end{aligned}
$$

i.e., $\nabla_{e_{1}} e_{2} \in \Gamma(\mathcal{E} M)$ and $\nabla_{f_{1}} f_{2} \in \Gamma(\mathcal{F} M)$. As the Levi-Civita connection is torsion free we have that $\mathcal{E} M$ and $\mathcal{F} M$ are involutive.

By the Theorem of Frobenius, these distributions define two foliations, such that, at each $p \in M$, there are two leaves $\mathcal{E}_{p}$ and $\mathcal{F}_{p}$ that intersect orthogonally at $p$. We want to understand what they are. The inclusions $i_{\mathcal{E}_{p}}$ and $i_{\mathcal{F}_{p}}$ of these leaves are immersions
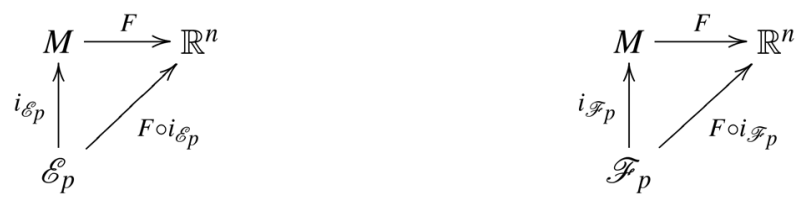

We need the symmetric (by Lemma 4.5) tensor

$$
(P * A)_{i j}:=P_{i}^{k} A_{k j} .
$$


Lemma 6.3. Under the hypothesis of Theorem 6.1, the following equations hold:

$$
\begin{gathered}
\theta^{k} \nabla_{k}^{\perp} A_{i j}=0, \\
A_{i j}=P_{i}^{k} A_{k j} .
\end{gathered}
$$

Proof. First, from (4.7) (with $\nabla \vec{H}=0$ ) and (6.3), we get

$$
\theta^{k} \nabla_{k}^{\perp}\left(P_{i}^{l} A_{l j}\right)=P_{i}^{l} A_{l j}-P_{i}^{l} A_{l j}=0 .
$$

To prove $(6.10)$, it is enough to show

$$
\left\|A_{ \pm}\right\|^{2}=\left\|P * A_{ \pm}\right\|^{2}
$$

One sees this using Equation (6.3) to calculate $\left\|A_{ \pm}-P * A_{ \pm}\right\|^{2}=\left\|A_{ \pm}\right\|^{2}-$ $\left\|P * A_{ \pm}\right\|^{2}$.

Let us then prove that $\left\|A_{ \pm}\right\|^{2}=\left\|P * A_{ \pm}\right\|^{2}$. First of all, using Equation (4.7) (with $\nabla_{i} \vec{H}=0$ ), Equation $(6.3)$ and $\theta^{k} \nabla_{k}^{\perp}\left(P_{i}^{l} A_{l j}\right)=0$, it holds that

$$
\theta^{k} \nabla_{k}\left(\left\|A_{ \pm}\right\|^{2}-\left\|P * A_{ \pm}\right\|^{2}\right)=-2\left(\left\|A_{ \pm}\right\|^{2}-\left\|P * A_{ \pm}\right\|^{2}\right)
$$

If $\theta=0$ at some point $p \in M$, then this equation implies $\left\|A_{ \pm}\right\|^{2}=\left\|P * A_{ \pm}\right\|^{2}$ and $A_{i j}=P_{i}^{l} A_{l j}$ at this point. So, without loss of generality, we can consider only the points $q \in M$ with $\theta(q) \neq 0$. Fix one of these and consider the integral curve $\gamma:(-a, b) \rightarrow M$ of $\theta$ with $\gamma(0)=q$, for some $a, b>0$. Along this curve, we define the function

$$
f(s):=\|\theta\|^{2}(\gamma(s))
$$

and get

$$
\frac{d}{d s} f=\nabla_{\dot{\gamma}}\|\theta\|^{2}=\theta^{k} \nabla_{k}\|\theta\|^{2}=2 \theta^{k} \theta^{l} \nabla_{k} \theta_{l}
$$

but, from $\vec{H}=-F^{\perp}$

$$
\nabla_{i} \theta_{j}=\nabla_{i}\left\langle F, F_{j}\right\rangle=g_{i j}-\left\langle\vec{H}, A_{i j}\right\rangle
$$

and $\theta^{i} \nabla_{i} \theta_{j}=\theta_{j}$ because of Equation (6.2), so that

$$
\frac{d}{d s} f=2 f
$$


This has a unique solution with $f(0)=\|\theta\|^{2}(q)$

$$
\|\theta\|^{2}(\gamma(s))=\|\theta\|^{2}(q) \mathrm{e}^{2 s}>0
$$

in particular $\|\theta\|^{2}(\gamma(s)) \neq 0$ for all $s \in(a, b)$, then these integral curves do not cross any singular point and the maximal integral curve is defined for all $\mathbb{R}$ and it is not closed (because of injectivity of $\mathrm{e}^{2 s}$ ). Over this same curve, we define functions $\tilde{f_{ \pm}}: \mathbb{R} \rightarrow \mathbb{R}$

$$
\tilde{f_{ \pm}}(s):=\left(\left\|A_{ \pm}\right\|^{2}-\left\|P * A_{ \pm}\right\|^{2}\right)(\gamma(s))
$$

and, using Equation (6.12), get $\frac{d \tilde{f_{ \pm}}}{d s}=-2 \tilde{f_{ \pm}}$. This has a unique solution with $\tilde{f_{ \pm}}(0)=\left(\left\|A_{ \pm}\right\|^{2}-\left\|P * A_{ \pm}\right\|^{2}\right)(q)$

$$
\left(\left\|A_{ \pm}\right\|^{2}-\left\|P * A_{ \pm}\right\|^{2}\right)(\gamma(s))=\left(\left\|A_{ \pm}\right\|^{2}-\left\|P * A_{ \pm}\right\|^{2}\right)(q) \mathrm{e}^{-2 t} .
$$

If $\left(\left\|A_{ \pm}\right\|^{2}-\left\|P * A_{ \pm}\right\|^{2}\right)(q) \neq 0$, then $\left(\left\|A_{ \pm}\right\|^{2}-\left\|P * A_{ \pm}\right\|^{2}\right)(\gamma(s)) \rightarrow \pm \infty$ as $s \rightarrow-\infty$ and this contradicts the boundedness of $\left\|A_{ \pm}\right\|^{2}$. So $A=P * A$ and (6.11) implies (6.9).

Let us now examine the leaves of the distribution $\mathcal{E} M$.

Lemma 6.4. Under the hypothesis of Theorem 6.1, it holds that $\mathcal{E}_{p}$ is immersed into $\mathcal{H}^{n-1}\left(\|F\|^{2}(p)\right)$ through $F \circ i_{\mathcal{E}_{p}}$. Beyond this, $\mathcal{E}_{p}$ is geodesically complete and there is $q \in M$ so that $F \circ i_{\mathcal{E}_{q}}$ is a minimal immersion into $\mathcal{H}^{n-1}\left(\|F\|^{2}(q)\right)$.

Proof. $\mathcal{E}_{p}$ is an $r$-dimensional manifold immersed in $M$ under the natural inclusion $i_{\mathcal{E}}$. Let $A_{F \circ i_{\mathcal{E}}}$ and $A_{i_{\mathcal{E}}}$ denote the second fundamental tensors of $F \circ i_{\mathcal{E}}$ and $i_{\mathcal{E}}$.

From Equation (3.4), it holds that

$$
A_{F \circ i_{\mathcal{E}}}=A_{F}+d F\left(A_{i_{\mathcal{E}}}\right) .
$$

On the other hand one can write, for local vector fields $e_{1}, e_{2} \in \Gamma\left(T \mathcal{E}_{p}\right)$

$$
A_{i_{\mathcal{E}}}\left(e_{1}, e_{2}\right)=\nabla_{e_{1}} e_{2}-\nabla_{e_{1}}^{\prime} e_{2},
$$

where $\nabla^{\prime}$ is the Levi-Civita connection of $\mathcal{E}_{p}$ (with respect to the induced metric). But $\nabla_{e_{1}} e_{2} \in \Gamma(\mathcal{E} M)$ by Equation (6.7) and $d i_{\mathcal{E}}\left(\nabla_{e_{1}}^{\prime} e_{2}\right) \in \Gamma(\mathcal{E} M)$, 
yet $A_{i_{\mathcal{E}}}\left(e_{1}, e_{2}\right) \in \Gamma\left(T \mathcal{E}^{\perp}\right)$, so

$$
A_{i_{\mathcal{E}}}=0
$$

Then, in particular, the geodesics of $\mathcal{E}_{p}$ are also geodesics of $M$ and, as $M$ is geodesically complete, so is $\mathcal{E}_{p}$. $V^{i} \frac{\partial}{\partial x^{i}}$

From Equation (6.6) we get, for any $q \in \mathcal{E}_{p}$ and all $V \in \mathcal{E} M_{i_{\mathcal{E}}(q)}, V=$

$$
0=\theta_{j} V^{j}=\left\langle F, F_{j}\right\rangle V^{j}=\langle F, d F(V)\rangle
$$

which means that $F\left(i_{\mathcal{E}}(q)\right) \in T_{q} \mathcal{E}_{p}^{\perp}$ (the normal space of $F \circ i_{\mathcal{E}}$ at $q$ ) and

$$
V\|F\|^{2}=2 V^{j}\left\langle F_{j}, F\right\rangle=2\langle d F(V), F\rangle=0
$$

so that $\|F\|^{2}$ is constant on the leaf $\mathcal{E}_{p}$ (but it depends on $p$ ), and $\mathcal{E}_{p}$ is immersed, through $F \circ i_{\mathcal{E}}$, in the hyperquadric $\mathcal{H}^{n-1}\left(\|F\|^{2}(p)\right)$.

Let us now take a look at a special leaf of the distribution $\mathcal{E} M$. There is a point $q \in M$, with $\|F(q)\|^{2}=\min _{x \in M}\|F(x)\|^{2}$ (because of the mainly positive assumption). Let us consider the leaf $\mathcal{E}_{q}$, which should be a minimal surface.

The norm of $F$ must be constant over this leaf by Equation (6.17), so that all the points of the leaf minimize the norm of $F$. But then, $2\langle d F(X), F\rangle=$ $X\|F\|^{2}=0$ for any $X \in T_{q^{\prime}} M, q^{\prime} \in i_{\mathcal{E}}\left(\mathcal{E}_{q}\right)$, this means that $F\left(q^{\prime}\right)$ is orthogonal to $T_{q^{\prime}} M$, i.e.

$$
F^{\perp}\left(q^{\prime}\right)=F\left(q^{\prime}\right)
$$

We claim that $F \circ i_{\mathcal{E}}\left(\mathcal{E}_{q}\right)$ is a minimal surface of the hyperquadric $\mathcal{H}^{n-1}$ $\left(\|F\|^{2}(q)\right)$. First, the Levi-Civita connection on the hyperquadric is given by the projection $\left(\operatorname{Pr}_{\mathcal{H}^{n-1}}\right)$ of the Levi-Civita connection of $\mathbb{R}^{q, n}$, which we denote $D$, over the tangent bundle of the hyperquadric. Then, using Equation (6.14) with $A_{i_{\mathcal{E}}}=0$, it holds

$$
\begin{aligned}
A_{\mathcal{H}^{n-1}}(X, Y) & =\operatorname{Pr}_{\mathcal{H}^{n-1}}\left(D_{X} Y\right)-\nabla_{X}^{\prime} Y=\operatorname{Pr}_{\mathcal{H}^{n-1}}\left(A_{F \circ i_{\mathcal{E}}}(X, Y)\right) \\
& =\operatorname{Pr}_{\mathcal{H}^{n-1}}\left(A_{F}(X, Y)\right) .
\end{aligned}
$$

On the other hand, take a vector $V \in T_{q^{\prime}} M^{\perp}, q^{\prime} \in \mathcal{E}_{q}$, then, using that $P^{i j} A_{i j}$ is in the same direction as $\vec{H}$ (Equation (4.17)), one gets $\left\langle P^{i j} A_{i j}\right.$, $V\rangle=0$. 
Take an orthonormal basis $\left\{e_{1}, \ldots, e_{r}, f_{1}, \ldots, f_{m-r}\right\}$ of $T_{q^{\prime}} M$ such that $\left\{e_{1}, \ldots, e_{r}\right\}$ is a basis of $\mathcal{E} M_{q^{\prime}}$ and $\left\{f_{1}, \ldots, f_{m-r}\right\}$ is a basis of $\mathcal{F} M_{q^{\prime}}$, then $\operatorname{tr}_{\mathcal{E}}\langle A, V\rangle=\sum_{i=1}^{r}\left\langle V, A\left(P\left(e_{i}\right), e_{i}\right)+\sum_{i=1}^{m-r}\left\langle V, A\left(P\left(f_{i}\right), f_{i}\right)\right\rangle=\operatorname{tr}_{M}\langle V, P * A\rangle=0\right.$ where we used that $P\left(e_{i}\right)=e_{i}$ and $P\left(f_{i}\right)=0$. This holds for any $q^{\prime} \in i_{\mathcal{E}}\left(\mathcal{E}_{q}\right)$ and means that $\operatorname{tr}_{\mathcal{E}} A=\mathfrak{a}(x) \vec{H}$ for some continuous function $\mathfrak{a}: \mathcal{E}_{q} \rightarrow \mathbb{R}$. By Equations (6.18) and (6.19) and denoting $\vec{H}_{\mathcal{H}^{n-1}}$ the mean curvature vector of the immersion of $\mathcal{E}_{q}$ into $\mathcal{H}^{n-1}\left(\|F\|^{2}(q)\right)$, we get at $q^{\prime}$

$$
\vec{H}_{\mathcal{H}^{n-1}}=\operatorname{Pr}_{\mathcal{H}^{n-1}}\left(\operatorname{tr}_{\mathcal{E}} A\right)=\operatorname{Pr}_{\mathcal{H}^{n-1}}(\mathfrak{a} \vec{H})=\operatorname{Pr}_{\mathcal{H}^{n-1}}\left(-\mathfrak{a} F^{\perp}\right)=\operatorname{Pr}_{\mathcal{H}^{n-1}}(-\mathfrak{a}(x) F)=0
$$

because the position vector is orthogonal to the hyperquadric. Then $\mathcal{E}_{q}$ is a minimal surface of the hyperquadric $\mathcal{H}^{n-1}(r)$, because $\|F\|^{2}(q)=\|\vec{H}\|^{2}(q)=$ $r$ by Equation (6.4).

We will now analyze the leaves of the distribution $\mathcal{F} M$.

Lemma 6.5. Under the hypothesis of Theorem 6.1, it holds that $F \circ i_{\mathcal{F}}\left(\mathcal{F}_{p}\right)$ is an affine space in $\mathbb{R}^{q, n}$ for any $p \in M$. Beyond that, if $q \in i_{\mathcal{E}}\left(\mathcal{E}_{p}\right)$, then $F \circ i_{\mathcal{F}}\left(\mathcal{F}_{p}\right)$ and $F \circ i_{\mathcal{F}}\left(\mathcal{F}_{q}\right)$ are parallel.

For a proof, see [26].

All that is left of Theorem 6.1 is showing that $F(M)$ is the product $F\left(\mathcal{E}_{q}\right) \times F\left(\mathcal{F}_{q}\right)$, where $q \in M$ minimizes $\|F\|^{2}$. This can be done considering the map $\mathfrak{h}$ defined as.

Let $q \in M$ be a minimal point of $\|F\|^{2}$ and $\left\{f_{1}, \ldots, f_{m-r}\right\}$ be an orthonormal basis of $\mathcal{F} M_{q}$. We define a function $\mathfrak{h}: \mathcal{E}_{q} \times \mathbb{R}^{m-r} \rightarrow F(M)$, given by

$$
\mathfrak{h}(p, X)=F\left(i_{\mathcal{E}}(p)\right)+X^{i} d F\left(f_{i}\right) \forall X=\left(X^{1}, \ldots, X^{m-r}\right) \in \mathbb{R}^{m-r}, p \in \mathcal{E}_{q}
$$

As all the leaves $\mathcal{F}_{q^{\prime}}, q^{\prime} \in \mathcal{E}_{q}$, are parallel, the image of $\mathfrak{h}$ is indeed contained in $F(M)$. Then $F(M)$ is the product of an affine space with a minimal surface of the hyperquadric $\mathcal{H}^{n-1}(r)$ with $\|\vec{H}\|^{2}=r$.

Remark 6.6. The induced (from $\mathbb{R}^{q, n}$ ) inner product on the affine space has to be positive definite, because $F$ is spacelike. 


\section{The second case}

Theorem 7.1. Let $M$ be a smooth manifold and $F: M \rightarrow \mathbb{R}^{q, n}$ be a mainly positive, spacelike, shrinking self-similar solution of the MCF with bounded geometry such that $F(M)$ is unbounded. Beyond that, let $F$ satisfy the conditions: $\|\vec{H}\|^{2}(p) \neq 0$ for all $p \in M$ and the principal normal is parallel in the normal bundle $\left(\nabla^{\perp} \nu \equiv 0\right)$. If $\nabla\|\vec{H}\|(p) \neq 0$ for some $p \in M$, then

$$
F(M)=\Gamma \times \mathbb{R}^{m-1},
$$

where $\Gamma$ is a rescaling of an Abresch 8 Langer curve in a spacelike plane and $R^{m-1}$ is an $m-1$ dimensional spacelike affine space in $\mathbb{R}^{q, n}$.

The proof of this theorem follows the same ideas of the first case (section 6 ) but with different equations. Now there are regions where $\nabla\|\vec{H}\|(p) \neq 0$ and in these regions the tensor $P_{i j}$ has only a non-zero eigenvalue, so the distribution $\mathcal{E} M$ is spanned by a vector field, which can be extended over the whole $M$ and the leaves of this distribution lie in planes. One of the leaves can be proven to be a solution to the curve shortening flow, thence a curve of Abresch and Langer. Some extra details can be found in [20, 27].

Remark 7.2. In particular, $M$ is contained in the product of an affine space and a plane, both spacelike, so that $\|\vec{H}\|^{2}>0$.

Remark 7.3. We found that there are no spacelike self-shrinkers of the $\mathrm{MCF}$ with timelike mean curvature in any of the treated cases, so that Theorems 4.2, 5.2, 5.7, 6.1 and Remark 7.2 sum up to:

Theorem 7.4. There are no spacelike self-shrinkers $F: M \rightarrow\left(\mathbb{R}^{n},\langle\cdot, \cdot\rangle\right)$ of the MCF that satisfy

- $F(M)$ unbounded and $F$ is mainly negative and has bounded geometry or

- $\|\vec{H}\|^{2}<0$ and one of the following:

(1) $M$ is compact.

(2) $F(M)$ is unbounded, $M$ is stochastic complete and $\sup _{M}\|F\|^{2} \leq \infty$. 
(3) $F(M)$ is unbounded, $F$ is mainly positive, has bounded geometry and the principal normal parallel in the normal bundle.

\section{Acknowledgments}

This work was done with financial support of the CNPq - National Counsel of Scientific and Technologic Development from Brazil - and developed at the Leibniz Universit at Hannover during the author's $\mathrm{PhD}$.

\section{References}

[1] U. Abresch and J. Langer, The normalized curve shortening flow and homothetic solutions, J. Differential Geom., 23(2) (1986), 175-196.

[2] M.R. Adames, Spacelike self-similar solutions of the mean curvature flow: in pseudo-Euclidean spaces, Sudwestdeutscher Verlag fur Hochschulschriften, Saarbrucken, Germany, 2012.

[3] H. Anciaux, Construction of Lagrangian self-similar solutions to the mean curvature flow in $\mathbb{C}^{n}$, Geom. Dedicata, 120 (2006), 37-48.

[4] S.B. Angenent, Shrinking doughnuts, (Gregynog, 1989), Progress in nonlinear differential equations and their applications, 7, Birkhäuser Boston, Boston, MA, 1992, 21-38.

[5] C. Baker, A partial classification of type I singularities of the mean curvature flow in high codimension, preprint (2011).

[6] M. Bergner and L. Schäfer, Time-like surfaces of prescribed anisotropic mean curvature in Minkowski space, (Dresden, 2010), Proc. 8th AIMS Conf., Discrete and Continous Dynamical Systems, 1, AIMS Press, Springfield, MO, 2011, 155-162.

[7] K.A. Brakke. The motion of a surface by its mean curvature, Mathematical Notes, 20, Princeton University Press, Princeton, NJ, 1978.

[8] H.-D. Cao and H. Li, A gap theorem for self-shrinkers of the mean curvature flow in arbitrary codimension, Calc. Var. Partial Differential Equations, 46(3-4) (2013), 879-889.

[9] D.L. Chopp, Computation of self-similar solutions for mean curvature flow, Experiment. Math., 3(1) (1994), 1-15. 
[10] B. Chow, P. Lu, and L. Ni. Hamilton's Ricci flow, Graduate Studies in Mathematics, 77, American Mathematical Society, Providence, RI, 2006.

[11] T.H. Colding and W.P. Minicozzi II, Generic mean curvature flow I: generic singularities, Ann. Math. (2), 175(2) (2012), 755-833.

[12] A.A. Cooper, A characterization of the singular time of the mean curvature flow, Proc. Amer. Math. Soc., 139(8) (2011), 2933-2942.

[13] Q. Ding and Z. Wang, On the self-shrinking systems in arbitrary codimensional spaces, preprint (2009).

[14] K. Ecker, Interior estimates and longtime solutions for mean curvature flow of noncompact spacelike hypersurfaces in Minkowski space, J. Differential Geom., 46(3) (1997), 481-498.

[15] K. Ecker and G. Huisken, Mean curvature evolution of entire graphs, Ann. Math. (2), 130(3) (1989), 453-471.

[16] - Parabolic methods for the construction of spacelike slices of prescribed mean curvature in cosmological spacetimes, Comm. Math. Phys., 135(3) (1991), 595-613.

[17] C. Gerhardt, Curvature flows in semi-Riemannian manifolds, Surveys in differential geometry. Vol. XII Geometric flows, Surveys in differential geometry, 12, International Press, Somerville, MA, 2008, 113-165.

[18] G. Huisken, Flow by mean curvature of convex surfaces into spheres, J. Differential Geom., 20(1) (1984), 237-266.

[19] - Asymptotic behavior for singularities of the mean curvature flow, J. Differential Geom., 31(1) (1990), 285-299.

[20] — Local and global behaviour of hypersurfaces moving by mean curvature, in 'Differential geometry: partial differential equations on manifolds' (Los Angeles, CA, 1990), Proc. Symp. Pure Mathematics, 54, American Mathematical Society, Providence, RI, 1993, 175-191.

[21] T. Ilmanen, Lectures on mean curvature flow and related equations, preprint, (1998).

[22] - Singularities of mean curvature flow of surfaces, preprint, (1995). 
[23] D. Joyce, Y.-I. Lee, and M.-P. Tsui, Self-similar solutions and translating solitons for Lagrangian mean curvature flow, J. Differential Geom., 84(1) (2010), 127-161.

[24] G. Li and I.M.C. Salavessa, Mean curvature flow and Bernstein-Calabi results for spacelike graphs, in 'Differential geometry', World Science Publication, Hackensack, NJ, 2009, 164-174.

[25] B. O'Neill. Semi-Riemannian geometry, in 'Pure and Applied Mathematics', 103, Academic Press, [Harcourt Brace Jovanovich, Publishers], New York, 1983. With applications to relativity.

[26] K. Smoczyk, Mean curvature flow in higher codimension - introduction and survey, Global Differential Geometry, Springer Proc. Mathematics, 17, Springer, Berlin, Heidelberg, 2012, 231-274.

[27] - Self-shrinkers of the mean curvature flow in arbitrary codimension, Int. Math. Res. Not., 48 (2005), 2983-3004.

[28] L. Wang, A Bernstein type theorem for self-similar shrinkers, Geom. Dedicata, 151 (2011), 297-303.

[29] M.-T. Wang, Lectures on mean curvature flows in higher codimensions, in 'Handbook of geometric analysis'. No. 1. Advanced Lectures in Mathematics (ALM), 7, International Press, Somerville, MA, 2008, $525-543$.

[30] B. White, Partial regularity of mean-convex hypersurfaces flowing by mean curvature, Int. Math. Res. Not., 4, 186 ff., approx. 8 pp. (electronic), 1994.

Departamento ACADÊMico de Matemática

UTFPR - CAMPUS CURITIBA

CuRItibA - PR 80230-901

BRAZIL

Email address: marcioadames@utfpr.edu.br

ReCEived July 20, 2013 
